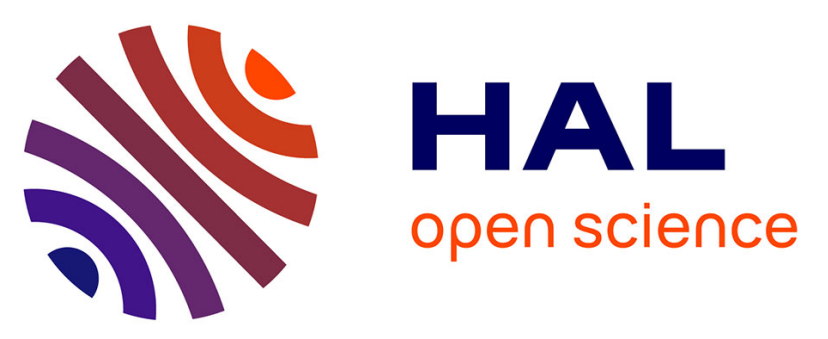

\title{
Effet de la matière organique produite dans les bassins de rétention des eaux pluviales sur la spéciation les éléments traces métalliques
}

Bruno J. Lemaire, Mathilde Lauzent, Viet Tran Khac, Gilles Varrault, Martin Seidl, Brigitte Vinçon-Leite

\section{To cite this version:}

Bruno J. Lemaire, Mathilde Lauzent, Viet Tran Khac, Gilles Varrault, Martin Seidl, et al.. Effet de la matière organique produite dans les bassins de rétention des eaux pluviales sur la spéciation les éléments traces métalliques. La Houille Blanche - Revue internationale de l'eau, 2015, 3 (3), pp.37-43. 10.1051/lhb/20150031 . hal-01238351

\section{HAL Id: hal-01238351 \\ https://hal-enpc.archives-ouvertes.fr/hal-01238351}

Submitted on 16 Nov 2017

HAL is a multi-disciplinary open access archive for the deposit and dissemination of scientific research documents, whether they are published or not. The documents may come from teaching and research institutions in France or abroad, or from public or private research centers.
L'archive ouverte pluridisciplinaire HAL, est destinée au dépôt et à la diffusion de documents scientifiques de niveau recherche, publiés ou non, émanant des établissements d'enseignement et de recherche français ou étrangers, des laboratoires publics ou privés. 


\title{
Effet de la matière organique produite dans les bassins de rétention des eaux pluviales sur la spéciation les éléments traces métalliques
}

\author{
Bruno Jacques LEMAIRE ${ }^{1,2}$, Mathilde LAUZENT ${ }^{1}$, Viet TRAN KHAC ${ }^{1}$, Gilles VARRAULT ${ }^{1}$, \\ Martin SEIDL ${ }^{1}$ et Brigitte VINÇON-LEITE
}

\author{
1. LEESU (UMR MA-102), Université Paris-Est, Ecole des Ponts ParisTech - 6-8 avenue Blaise Pascal, Cité Descartes, Champs sur Marne 77 \\ 455 Marne-La-Vallée Cedex 2, France-bruno.lemaire@leesu.enpc.fr \\ 2. AgroParisTech - 16 rue Claude Bernard F-75231 Paris Cedex 05, France
}

\begin{abstract}
RÉSUMÉ. - Eléments du réseau d'assainissement pluvial, les bassins de rétention en eau ont un fonctionnement hydrodynamique spécifique, proche de celui d'un étang. Le temps de séjour de l'eau y est plus élevé que dans les conduites, favorisant la sédimentation des particules. Pendant le printemps et l'été, des phases de mélange et de stratification thermique alternent. Une biomasse élevée de phytoplancton peut alors se développer et la matière organique qu'elle produit peut interagir avec les micropolluants. Ainsi, pour mieux comprendre le cycle biogéochimique des éléments traces métalliques (ETM), il paraît important de connaître l'effet de la matière organique produite dans les bassins de rétention sur les ETM.

Pour cela, nous avons étudié la spéciation du nickel, du cuivre, du zinc et du plomb dans l'eau dans un bassin de rétention en eau de Seine-Saint-Denis situé en dérivation d'une rivière canalisée qui l'alimente lors des fortes pluies. Les concentrations de temps sec en ETM totaux, dissous et labiles ont été déterminées. Les paramètres globaux ont également été mesurés. Par temps sec, peu de métaux labiles, potentiellement biodisponibles, sont présents dans le bassin (moins de $50 \%$ de la concentration totale), moins que dans la rivière (autour de $70 \%$ ). Par ailleurs, la concentration en chlorophylle est plus élevée dans le bassin $(90 \mathrm{à} 140 \mu \mathrm{g} / \mathrm{L})$ que dans la rivière $(30 \mu \mathrm{g} / \mathrm{L})$ : la matière organique dissoute autochtone semble favoriser la complexation des métaux dissous dans le bassin. La réduction de la biodisponibilité du nickel et du cuivre dans le bassin de rétention par rapport à la rivière qui l'alimente, qui devra être confirmée sur la base d'un plus grand nombre de mesures, montre l'intérêt de faire passer les eaux pluviales urbaines dans des bassins de rétention en eau pour réduire leur toxicité avant rejet dans le milieu récepteur.
\end{abstract}

Mots-clés : eutrophisation, biomasse de phytoplancton, temps sec

\section{Impact of the organic matter produced in stormwater retention ponds on trace metal speciation}

\begin{abstract}
Retention ponds, which are part of the stormwater drainage system, have specific hydrodynamics. The residence time of the water, higher than in the conduits, favours the sedimentation of particles. During spring and summer, mixing and thermal stratification alternate. A high phytoplankton biomass can develop and produce organic matter which can interact with micropollutants. That is why it is essential to understand the impact of organic matter locally produced on trace metals. For this purpose, we studied the speciation of nickel, copper, zinc and lead in the waters of a retention pond located in derivation of a channelled river in a Paris suburb (France). Three fractions of trace metals (total, dissolved and labile fractions) were sampled during dry weather and analyzed. During dry weather, few labile metals, potentially bioavailable, were present in the pond (less than $50 \%$ of the total concentration), less than in the river (40-70\%). The chlorophyll a concentration was higher in the pond (90 to $140 \mu \mathrm{g} / \mathrm{L})$ than in the river $(30 \mu \mathrm{g} / \mathrm{L})$ : autochthonous dissolved organic matter seems to favour the complexation of dissolved metals. The reduction of nickel and copper trace metal bioavailability in the retention pond compared to the river which feeds the pond, which needs to be confirmed with more measurements, shows the interest to have urban storm waters transit through retention ponds to reduce their toxicity before their release in the natural waters.
\end{abstract}

Key-words: eutrophication, phytoplankton biomass, dry weather

\section{INTRODUCTION}

Avec l'expansion urbaine, les systèmes classiques basés sur les réseaux se sont révélés insuffisants pour assurer l'assainissement pluvial des agglomérations. Les aménageurs on dû créer des bassins qui retiennent les eaux pendant la pluie et les évacuent ensuite à faible débit vers l'exutoire, si possible après une dépollution partielle par décantation des particules. Il existe plusieurs types de bassins de rétention des eaux pluviales : les bassins en eau, enterrés, secs, les bassins de décantation... [STU - Agences de l'eau 1994].

Depuis longtemps, parmi ces différents types de bassins, les bassins de décantation sont connus pour permettre la dépollution des eaux grâce à un long temps de séjour qui 
permet une bonne sédimentation des particules. Ils sont largement utilisés pour traiter les eaux de ruissellement pluvial fortement polluées avant leur rejet dans le milieu naturel (autoroutes, pistes d'aéroports, grands parkings, etc.). Ainsi, de nombreuses études in situ et en laboratoire ont porté sur le processus de sédimentation et ont montré son importance dans la dépollution des eaux pluviales dans les bassins [Yousef et al., 1996, Lee et al., 1997, Weiss et al., 2006, Karlsson et al. 2008 et 2010]. Les particules fines, entrâ̂nées par le ruissellement pluvial, servent de site d'adsorption aux micropolluants comme les éléments traces métalliques (ETM). La vitesse de sédimentation élevée de ces particules est favorable à une bonne décantation [Bressy 2010].

Les bassins de rétention en eau font quant à eux l'objet d'une demande sociale croissante. En plus de leur fonction hydraulique principale, ils ont souvent été aménagés pour permettre des activités de loisir, comme la promenade ou la pêche, et présentent ainsi un grand intérêt pour les citadins [Tassin et al., 2004]. Aujourd'hui, les collectivités cherchent à accrôtre la biodiversité de ces milieux aquatiques artificiels. C'est le cas du site d'étude présenté dans cet article, le bassin de rétention de Savigny, intégré dans le parc départemental du Sausset dans une zone Natura 2000 [Inventaire national du patrimoine naturel, 2006].

Le devenir des ETM dans un bassin de rétention en eau dépend de leur spéciation. La concentration totale d'un ETM dans le milieu, agrégeant la fraction particulaire et la fraction dissoute, est un mauvais indicateur de sa toxicité [Varrault et al., 2012]. Il faut considérer la fraction biodisponible, c'est-à-dire susceptible d'entrer en contact et/ou de contaminer les organismes [Gourlay-Francé et al., 2010]. Comme celle-ci dépend des organismes, on prend comme indicateur la fraction labile, qui rassemble les métaux libres, c'est-à-dire non complexés, et les complexes métalliques labiles. Ces complexes s'établissent entre les ETM et les différents ligands minéraux (chlorures, nitrates, carbonates,...) ou organiques (matière organique d'origine naturelle ou anthropique) présents dans le milieu. Tout comme les métaux libres, les complexes labiles, qui se dissocient facilement, sont potentiellement biodisponibles, tandis que les complexes inertes, qui se dissocient moins facilement, sont réputés peu ou pas biodisponibles. Généralement, les ligands minéraux forment des complexes labiles avec les ETM tandis que la matière organique forme plutôt des complexes inertes. Beaucoup d'auteurs s'intéressent uniquement à la contamination en ETM des sédiments des bassins de rétention et à la spéciation des métaux dans ces sédiments [Hossain et al., 2007, Stead-Dexter et al., 2004, El Mufleh et al., 2010, Clozel, 2006]. Peu d'études ont porté sur la spéciation des métaux dans l'eau des bassins de rétention pour évaluer leur biodisponibilité pour les organismes vivants.

Par le phénomène de biosorption, des organismes vivants accumulent des micropolluants présents dans l'eau de manière passive sur leur structure cellulaire externe [Chojnacka, 2010]. Une étude faite sur le phytoplancton du lac Novosibirskoye en Russie a montré que la biosorption joue sur la biodisponibilité et la toxicité des métaux. Le phytoplancton a continué d'y croître en présence de fortes concentrations de métaux dans l'eau (concentrations en cuivre de $500 \mu \mathrm{g} / \mathrm{L}$, en zinc de $250 \mu \mathrm{g} / \mathrm{L}$ en cadmium de $50 \mu \mathrm{g} / \mathrm{L}$ ), après une période initiale d'inhibition puis de sélection d'espèces tolérantes. La biosorption sur le phytoplancton a modifié la spéciation des métaux et réduit la fraction dissoute, la plus biodisponible et la plus toxique [Smolyakov et al., 2010]. Ainsi, la biosorption pourrait contribuer à la dépollution des eaux pluviales, mais elle reste peu étudiée dans cette optique.
Les bassins de rétention en eau ont un fonctionnement spécifique qui s'apparente à celui d'un petit lac urbain. Ils sont souvent de petite taille, peu profonds et répondent rapidement aux fluctuations météorologiques. Ils sont particulièrement sensibles aux changements de température de l'air. Ainsi, durant le printemps et l'été des phases de mélange et de stratification de la colonne d'eau alternent. Ces alternances et l'apport de nutriments par ruissellement urbain lors d'évènements pluvieux, peuvent favoriser le développement de phytoplancton [Tran Khac et al., 2012]. Celui-ci produit de la matière organique qui peut interagir avec les micropolluants métalliques ou organiques, directement ou après dégradation par les bactéries. De plus, le temps de séjour de l'eau, plus élevé que dans les autres ouvrages d'assainissement, favorise la sédimentation des particules et permet le changement de la répartition des ETM entre fractions particulaire et dissoute ainsi que leur changement de spéciation au sein de la phase dissoute ce qui peut modifier leur biodisponibilité.

Dans ce travail, nous nous sommes intéressés à l'effet de la matière organique produite dans un bassin de rétention en eau sur la spéciation des ETM. Nous avons tout d'abord étudié la spéciation de quelques métaux traces caractéristiques des eaux de ruissellement urbain $(\mathrm{Cu}, \mathrm{Zn}, \mathrm{Pb}, \mathrm{Cd}$ et $\mathrm{Ni})$, présents dans un bassin par temps sec. L'article présente la méthode d'échantillonnage par temps sec, la technique de spéciation des métaux ainsi que le suivi des paramètres globaux dans le bassin de rétention, et les concentrations et la spéciation des métaux mesurées par temps sec dans le bassin et dans la rivière qui l'alimente lors des fortes pluies.

\section{MATÉRIELS ET MÉTHODES}

\section{II.1. Site d'etude}

Le bassin de rétention en eau de Savigny se situe dans le parc départemental du Sausset à Aulnay-sous-Bois (Seine-Saint-Denis). La surface du plan d'eau permanent est de $4.3 \mathrm{ha}$, sa profondeur moyenne est de $1.5 \mathrm{~m}$, soit un volume permanent de $64000 \mathrm{~m}^{3}$. Lors d'un remplissage, la hauteur d'eau peut s'accroître de $1.4 \mathrm{~m}$ pour stocker jusqu'à $80000 \mathrm{~m}^{3}$ supplémentaires, grâce à la faible pente des berges. La situation géographique du bassin de Savigny l'amène à recevoir des eaux potentiellement chargées en ETM : eaux issues du ruissellement urbain (autoroute A104, ZAC Paris Nord II...), eaux pluviales issues des lagunes d'épuration de l'aéroport Roissy-Charles-de-Gaulle. Ainsi, ce site nous permet d'étudier le devenir des ETM dans un bassin de rétention en eau.

Le bassin de Savigny est alimenté par la nappe phréatique de Saint-Ouen et le ruisseau canalisé du Sausset. Le débit du ruisseau du Sausset est limité en aval du bassin par une vanne. Lors d'épisodes pluvieux intenses, le ruisseau alimente le bassin de Savigny par deux déversoirs situés en amont et en aval sur le Sausset. Les eaux s'écoulent par deux prébassins prévus pour limiter l'envasement du bassin de rétention. Hors épisodes de pluie exceptionnels, les écoulements entrant et sortant passent par le seul prébassin aval (PAV), dont le seuil est le plus bas (Figure 1).

\section{II.2. Campagnes de mesure}

\section{II.2.1. Stratégie d'échantillonnage}

Pour connaître les concentrations en ETM dans le bassin en absence de déversement du Sausset, deux campagnes de 


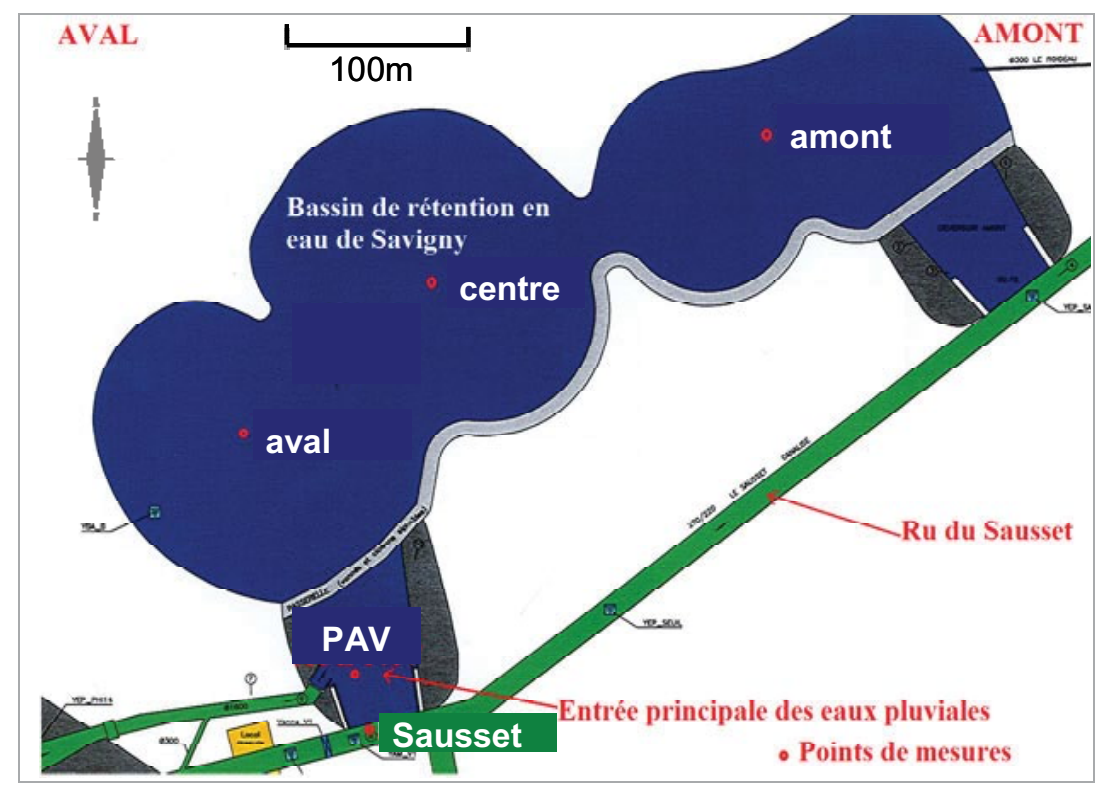

Figure 1 : Situation du bassin de rétention en eau de Savigny et localisation des points d'échantillonnage (PAV: prébassin aval ; source : DEA 93).

temps sec ont été organisées. Trois points d'échantillonnage ont été choisis dans le bassin : " amont » vers le prébassin amont, " centre » au centre du bassin de rétention, «aval " vers le prébassin aval. Un point est situé dans le prébassin aval («PAV »). Un dernier point d'échantillonnage est situé dans le ru du Sausset au droit du prébassin aval (Figure 1). Les échantillons ont été prélevés sous la surface.

\section{II.2.2. Paramètres mesurés in situ}

Aux trois points de mesure situés dans le bassin de Savigny, des profils de sonde sur toute la hauteur d'eau ont été réalisés. La température, la concentration en oxygène dissous, la conductivité et le $\mathrm{pH}$ sont déterminés avec une sonde multi-paramètres (Sea-Bird SBE 19-03). Les profils de concentrations en chlorophycées, cyanobactéries, diatomées, cryptophycées et la concentration totale en chlorophylle sont obtenus avec une sonde fluorimétrique (BBE-Moldaenke Fluoroprobe 3). La transparence de l'eau est mesurée au disque de Secchi.

\section{II.2.3. Analyses au laboratoire}

Trois fractions métalliques, totale, dissoute et inerte, sont mesurées sur les échantillons d'eau du Sausset et du bas$\sin$. La concentration totale en métaux est obtenue après la digestion des échantillons bruts, conservés à $\mathrm{pH} 2\left(\mathrm{HNO}_{3}\right.$ Optima), par une attaque acide par $\mathrm{HNO}_{3}$ (Optima, $65 \%$ ) et $\mathrm{HCl}$ (Optima, $30 \%)$ à chaud $\left(2 \mathrm{~h} 30\right.$ à $95^{\circ} \mathrm{C}$, système DigiPREP) qui permet l'extraction des métaux liés aux particules. Les échantillons sont filtrés avant analyse sur un filtre en téflon $(0.45 \mu \mathrm{m})$. La fraction dissoute est obtenue par filtration d'eau brute non acidifiée sur un filtre en acétate de cellulose $(0.45 \mu \mathrm{m})$. La fraction inerte des ETM est obtenue par le passage de ce filtrat à travers un disque chélatant (EMPORETM Chelex 100"). Constitué de polymères (polystyrène divinylbenzène) portant des groupements chélatants iminodiacétiques, il permet de séparer les métaux dissous labiles, incluant les métaux libres, retenus par le disque, d'une part, de la fraction inerte, d'autre part
[Varrault et al., 2012, Bracmort, 2010]. Les fractions dissoute et inerte sont conservées à $\mathrm{pH}$ inférieur à $2\left(\mathrm{HNO}_{3}\right.$ Optima) jusqu'à l'analyse.

Les fractions totale, dissoute et inerte sont ensuite analysées par spectrométrie de masse couplée à un plasma inductif (ICP-MS). Les ETM dont les concentrations ont été étudiées sont le nickel, le cuivre, le cadmium, le plomb et le zinc. Ils sont tous toxiques pour les organismes vivants, mais le cuivre et le zinc sont essentiels, contrairement au cadmium, au plomb et au nickel. Les limites de quantification de l'ICP-MS sont très basses, de $0.01 \mu \mathrm{g} / \mathrm{L}$ pour $\mathrm{Cd}, \mathrm{Pb}, \mathrm{Ni}$, $\mathrm{Cu}$ et $0.05 \mu \mathrm{g} / \mathrm{L}$ pour le $\mathrm{Zn}$. L'incertitude de mesure est de l'ordre de $10 \%$. La fraction labile est obtenue par différence entre la fraction dissoute et la fraction inerte.

De plus, nous avons mesuré les concentrations de différents paramètres permettant de caractériser les interactions de la matière organique avec les métaux : carbone organique dissous (COD), carbone organique particulaire (COP), matières en suspension (MES), chlorophylle a, et les nutriments permettant la production phytoplanctonique dans le bassin : nitrates, phosphates et phosphore total.

\section{RÉSULTATS}

Nous avons caractérisé la qualité de l'eau du bassin Savigny par temps sec grâce à deux campagnes réalisées le $21 / 02 / 2013$ et le $25 / 04 / 2013$. Les derniers remplissages partiels du bassin de rétention par débordement du Sausset qui ont précédé ces campagnes représentaient un volume de $1500 \mathrm{~m}^{3}$ environ chacun, et avaient eu lieu respectivement environ un mois et quinze jours auparavant.

\section{III.1. Paramètres globaux}

Les concentrations des paramètres globaux des deux campagnes de temps sec $(21 / 02 / 2013$ et $25 / 04 / 2013)$ sont présentées dans le Tableau 1. 
Tableau 1 : Paramètres globaux lors des deux campagnes de temps sec (matières en suspension (MES), carbone organique particulaire (COP), carbone organique dissous (COD), nitrates, phosphore total $\left(P_{t}\right)$, phosphates, chlorophylle a).

\begin{tabular}{|c|c|c|c|c|c|c|c|c|}
\hline Date & Point & $\begin{array}{c}\text { MES } \\
(\mathrm{mg} / \mathrm{L})\end{array}$ & $\begin{array}{c}\mathrm{COP} \\
(\mathrm{mgC} / \mathrm{L})\end{array}$ & $\begin{array}{c}\text { COD } \\
(\mathrm{mg} / \mathrm{L})\end{array}$ & $\begin{array}{c}\mathrm{NO}_{3}^{-} \\
(\mathrm{mgN} / \mathrm{L})\end{array}$ & $\begin{array}{c}P_{t} \\
(\mu g P / L)\end{array}$ & $\begin{array}{c}\text { phosphates } \\
(\mu \mathrm{gP} / \mathrm{L})\end{array}$ & $\begin{array}{c}\text { Chlorophylle a } \\
(\mu \mathrm{g} / \mathrm{L})\end{array}$ \\
\hline \multirow{5}{*}{$21 / 02 / 2013$} & Sausset & - & - & - & - & - & - & - \\
\hline & Amont & 23.6 & 8.2 & 2.1 & 0.36 & 153 & 17 & 87 \\
\hline & Centre & 28.9 & 9.3 & 2.2 & 0.30 & 173 & 17 & 111 \\
\hline & Aval & 26.0 & 10.0 & 2.2 & 0.28 & 202 & 43 & 110 \\
\hline & PAV & - & - & - & - & - & - & - \\
\hline \multirow{5}{*}{$25 / 04 / 2013$} & Sausset & 17.5 & 3.4 & 3.1 & 3.80 & 129 & 2 & 29 \\
\hline & Amont & 39.5 & 11.3 & 4.3 & 0.03 & 321 & 12 & 107 \\
\hline & Centre & 38.5 & 11.1 & 4.1 & 0.04 & 242 & 9 & 108 \\
\hline & Aval & 51.7 & 13.6 & 4.3 & 0.03 & 319 & 17 & 136 \\
\hline & PAV & 46.0 & 13.2 & 4.5 & 0.02 & 300 & 29 & 148 \\
\hline
\end{tabular}

La concentration en chlorophylle augmente entre les deux campagnes dans le bassin de rétention, elle est comprise entre 87 et $110 \mu \mathrm{g} / \mathrm{L}$ le $21 / 02 / 13$ et entre 107 et $136 \mu \mathrm{g} / \mathrm{L}$ le 25/04/13 (points amont, centre et aval). On observe une légère baisse de la concentration en chlorophylle de l'aval vers l'amont du bassin de rétention lors des deux campagnes. La concentration en chlorophylle dans le Sausset n'a été mesurée que le 25/04/2013 ; à cette date elle est nettement plus faible que dans le bassin $(29 \mu \mathrm{g} / \mathrm{L})$.

Lors de la campagne du 21/02/2013, une sursaturation en oxygène de 115 à $118 \%$ selon les points est mesurée, indiquant une forte activité photosynthétique du phytoplancton présent dans le bassin. Le $\mathrm{pH}$ élevé, de l'ordre de 8.6, traduit également une forte activité photosynthétique. Lors de la seconde campagne du 25/04/13, la sursaturation est encore plus forte. Elle décroît de $180 \%$ à la surface à $120 \%$ vers le fond.

\section{III.2. Métaux}

Les concentrations en nickel, cuivre, plomb et zinc totaux des deux campagnes de temps sec sont présentées sur la Figure 2. Pour ces deux campagnes, les concentrations totales de ces métaux sont très variables entre les points de mesure dans le bassin et dans le ru du Sausset. Elles sont comprises entre 2.5 et $7.7 \mu \mathrm{g} / 1$ pour le nickel, 1.5 et $3.8 \mu \mathrm{g} / 1$ pour le cuivre, entre 6.1 et $30 \mu \mathrm{g} / 1$ pour le zinc et entre 1.4 et $2.3 \mu \mathrm{g} / \mathrm{l}$ pour le plomb. Les concentrations en cadmium restent toujours en dessous ou proches de la limite de quantification $(0.01 \mu \mathrm{g} / \mathrm{L})$ et ne sont pas présentées.

Les concentrations en ETM dissous observées dans le bassin de rétention (moyenne sur les deux campagnes et les points amont, centre et aval) et dans le ru du Sausset ( $2^{\mathrm{e}}$ campagne), sont largement au-dessous des normes de qualité environnementales en moyenne annuelle (NQE-MA) [MEDDE, 2012] hors fond géochimique pour le cadmium (non présenté), le nickel et le plomb (Figure 3). Par contre, les concentrations en cuivre et zinc sont proches de la norme dans le bassin de rétention. Pour ces deux éléments, les concentrations observées dans le ru du Sausset la dépassent largement.

La spéciation des ETM, mesurée lors de la campagne du $25 / 04 / 2013$, varie elle aussi en fonction des points, comme le montre la Figure 4. Les ETM dissous sont principalement sous forme inerte aux points aval, centre et amont, dans le bassin de rétention. La spéciation diffère entre le bassin de rétention et le Sausset pour le nickel et le cuivre dont la fraction labile est pratiquement absente dans le bassin. Le zinc dissous est majoritairement sous forme inerte dans le Sausset et le bassin de rétention. En ce qui concerne le plomb, la fraction dissoute est pratiquement absente dans le Sausset comme dans le bassin.
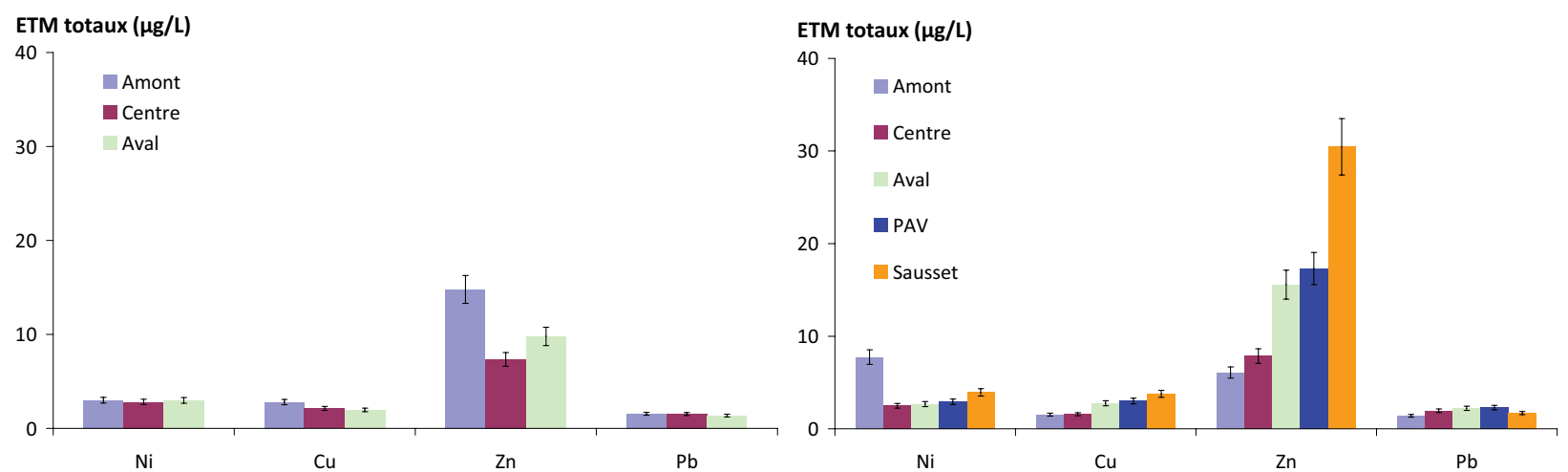

Figure 2 : Concentrations en nickel, cuivre, plomb et zinc totaux des deux campagnes de temps sec (à gauche, 21/2/2013 et à droite, 25/4/2013). 


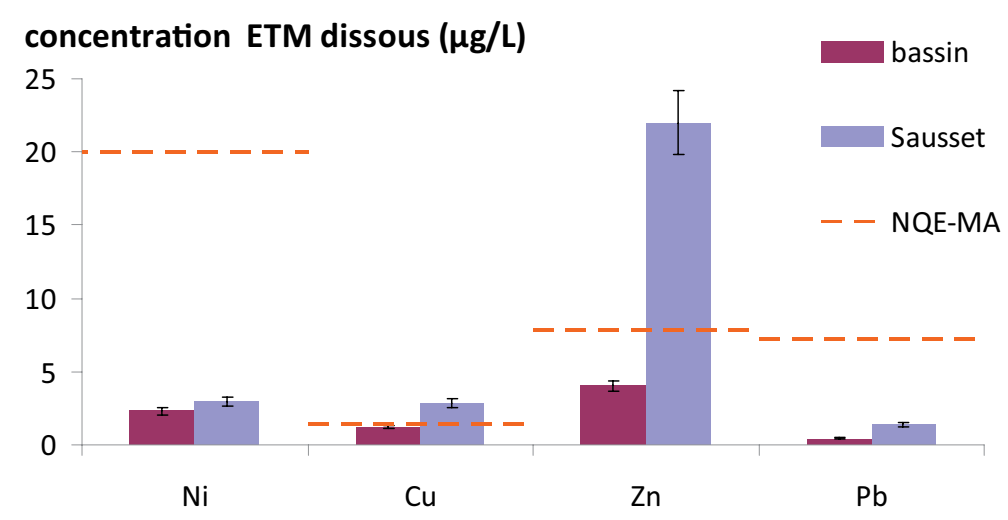

Figure 3 : Concentrations en $\mathrm{Ni}, \mathrm{Cu}, \mathrm{Zn}$ et $\mathrm{Pb}$ dissous dans le bassin de rétention (moyenne sur les points amont, centre et aval et sur les deux campagnes de temps sec) et dans le ru du Sausset (campagne du 25/04/2013) et normes de qualité environnementale en moyenne annuelle (NQE-MA), hors fond géochimique.
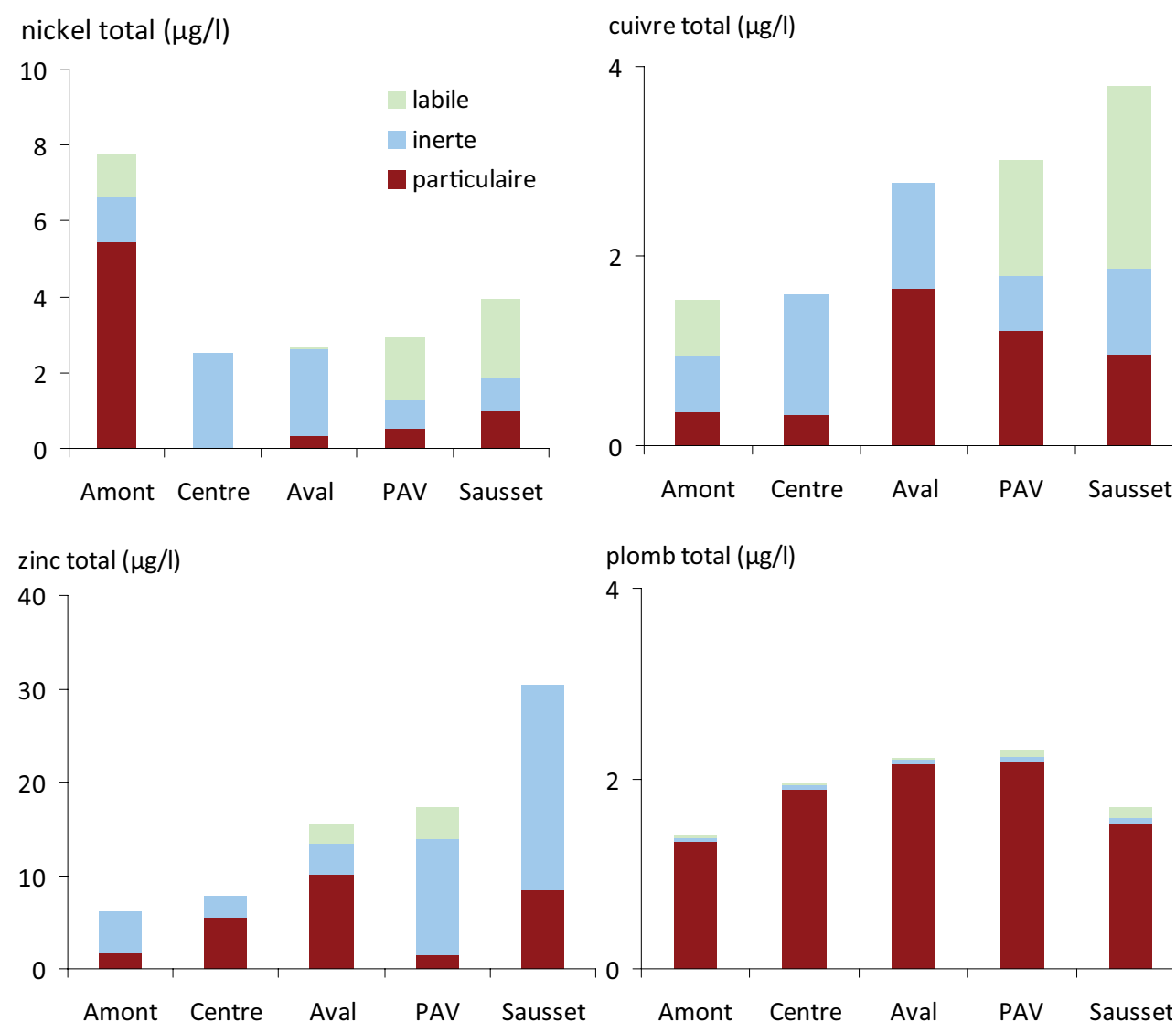

plomb total $(\mu \mathrm{g} / \mathrm{l})$

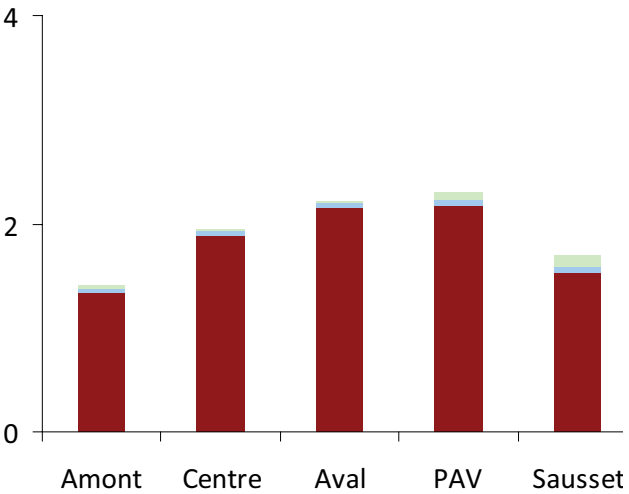

Figure 4 : Campagne du 25/04/2013 - variation de la concentration totale en nickel, cuivre, zinc et plomb et de sa répartition entre fractions labile, inerte et particulaire aux différents points de mesures du bassin de rétention et du ru du Sausset.

La Figure 5 présente le rapport entre les fractions labile et dissoute pour le nickel, le cuivre, le zinc et le plomb. Ce pourcentage (inférieur à $50 \%$ ) est beaucoup plus faible pour les points situés dans le bassin de rétention que dans le Sausset (environ $70 \%$ ). Pour le zinc qui ne présente pas de fraction labile dans le Sausset, aucune conclusion ne peut être tirée de cette campagne. Le bassin de rétention semble réduire la biodisponibilité de ces micropolluants métalliques, car la fraction labile est un indicateur de la fraction biodisponible.

\section{DISCUSSION ET CONCLUSION}

Nous avons comparé nos mesures avec des mesures plus anciennes. En 1989, des mesures de concentration en plomb, zinc et cadmium dans les eaux transitant dans des réseaux séparatifs et unitaires ont été effectuées sur six sites d'études, dont le bassin de Savigny, intégré dans le réseau séparatif de la commune d'Aulnay-sous-Bois [Flores-Rodriguez, 1989]. Nous avons comparé les données 


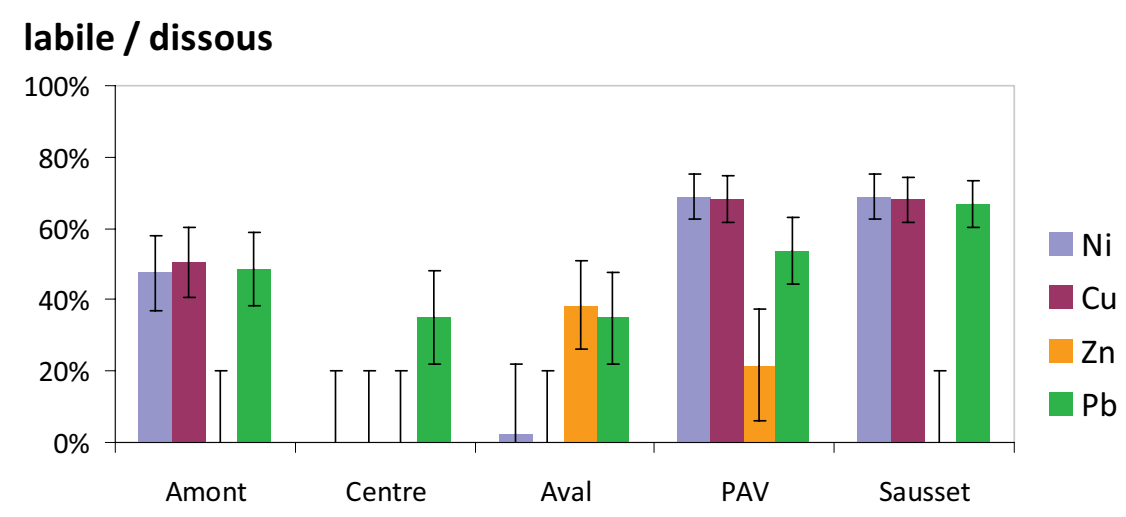

Figure 5 : Pourcentage de la fraction labile par rapport à la totalité du dissous pour le nickel, le cuivre, le zinc et le plomb lors de la campagne du 25/04/2013.

pour le plomb, le zinc et le cadmium. Les concentrations en plomb dissous ont nettement diminué de $3.05 \pm 0.72 \mu \mathrm{g} / \mathrm{L}$ en 1989 à $0,07-0.127 \pm 0.01 \mu \mathrm{g} / \mathrm{L}$ en 2013 dans la partie « amont » du bassin. Dans le prébassin aval, elles ont également diminué de $10.5 \mu \mathrm{g} / \mathrm{L}$ en 1989 à environ $0.12 \pm 0.01$ $\mu \mathrm{g} / \mathrm{L}$ en 2013. La diminution de la concentration en plomb peut s'expliquer par l'arrêt de l'utilisation du plomb comme additif dans les essences, appliqué en France métropolitaine depuis le 2 janvier 2000 (Miquel, 2001). La concentration en cadmium dissous a également baissé pendant cette période. Elle est maintenant en dessous des limites de quantification $(0.01 \mu \mathrm{g} / \mathrm{L})$ au point amont et à environ $0,015 \mu \mathrm{g} / \mathrm{L}$ dans le prébassin aval. Par contre la concentration en zinc dissous a nettement augmenté de $2.4 \mu \mathrm{g} / \mathrm{L}$ dans le prébassin aval en 1989 à $15.3 \pm 1.5 \mu \mathrm{g} / \mathrm{L}$ en 2013 . Dans la partie « amont» du bassin de rétention, en 1989 , la concentration était de $3.5 \mu \mathrm{g} / \mathrm{L}$ alors qu'elle est de $4.7 \pm 0.5 \mu \mathrm{g} / \mathrm{L}$ en 2013. Cette augmentation peut être due à la corrosion des toitures, à l'augmentation du trafic routier ou encore à un rejet particulier sur le bassin versant.

Sur la base de nos deux campagnes de temps sec, l'étang de Savigny peut être classé hypereutrophe selon la classification de Vollenweider [Vollenweider, 1982]: la transparence de l'eau est inférieure à $1 \mathrm{~m}$, la concentration en phosphore total est supérieure à $100 \mu \mathrm{g} / \mathrm{L}$, la concentration en chlorophylle est supérieure à $25 \mu \mathrm{g} / \mathrm{L}$, le $\mathrm{pH}$ est élevé $(8.5$ à 9.7 lors des deux campagnes) et le lac est sursaturé en oxygène (115 à $180 \%)$.

D'après les résultats de la campagne de temps sec du $25 / 04 / 13$, il semble que la production phytoplanctonique a un effet sur la spéciation des métaux. Les valeurs élevées de chlorophylle, MES et $\mathrm{pH}$ sont sous la dépendance du développement algal qui constitue l'essentiel des MES, lesquelles constituent des sites d'adsorption des métaux. Lors de cette campagne, la fraction inerte est plus importante pour le nickel, le cuivre, et le plomb dans le bassin de Savigny que dans le Sausset. Ce n'est pas le cas pour le zinc, majoritairement sous forme inerte dans le Sausset comme dans le bassin de rétention, résultat inattendu car le zinc n'est pas réputé pour former des complexes stables. La réduction de la fraction labile, potentiellement biodisponible, dans le bassin de rétention, qui devra être validée par d'autres campagnes, est à rapprocher des résultats de Pernet-Coudrier (2008) et Matar (2012), qui ont montré que la matière organique dissoute urbaine diminue la biodisponibilité des métaux. Le phytoplancton et les bactéries excrètent de la matière organique autochtone colloïdale, avec laquelle les métaux dissous peuvent se complexer. Comme la matière organique urbaine, cette matière organique d'origine aquatique produite dans le bassin de rétention présente de nombreux groupements fonctionnels fixateurs de métaux et notamment des groupements azotés. Par ailleurs, le phytoplancton, en consommant du dioxyde de carbone pour la photosynthèse, entraîne une augmentation $\mathrm{du} \mathrm{pH}$, qui modifie à son tour les équilibres de solubilité des métaux (Meybeck et al. 1998).

La réduction de la biodisponibilité du nickel et du cuivre dissous dans le bassin de rétention, qui devra être confirmée sur la base d'un plus grand nombre de mesures, montre l'intérêt de faire passer les eaux pluviales dans des bassins de rétention en eau pour réduire leur toxicité avant rejet dans le milieu récepteur. Ce séjour entraînera par ailleurs la décantation d'une partie des particules et des ETM qui y sont liées, ce qui suppose de surveiller la contamination des sédiments et leur remise en suspension sous l'effet de la bioturbation, du vent ou lors des remplissages.

La suite du travail portera sur la comparaison des résultats des analyses des différentes fractions métalliques sur les échantillons prélevés par temps de pluie et par temps sec, afin de mettre en évidence d'éventuelles différences de la spéciation des métaux et le rôle joué par le bassin de rétention.

\section{REMERCIEMENTS}

Les auteurs remercient Mme Boudahmane, M. Saad et M. Dubois du LEESU pour leur support technique dans la réalisation des campagnes de terrain et pour les analyses au laboratoire, et M. Daniel Thévenot et Mme Lise Fechner-Guieu pour leurs suggestions. Ils remercient également Mme Boulay, M. Bazot, M. Bovero, M. Braun, M. Desetables, M. Oudin, M. Queinec et M. Vandelannoote de la Direction de l'eau et de l'assainissement du département de Seine-Saint-Denis (DEA 93), M. Gibaud et M. Vauray de la Direction de la nature, des paysages et de la biodiversité (DNPB 93) pour le temps qu'ils nous ont accordé, sans lequel ce travail n'aurait pas pu être réalisé. Enfin, les auteurs remercient pour leur contribution financière les partenaires opérationnels du programme de recherche Observatoire des polluants urbains (OPUR) dans le cadre duquel cette recherche a été effectuée, AESN, SIAAP, Mairie de Paris, CG92, CG93, CG94. 


\section{REFERENCES}

Bracmort G. (2010) - Détermination des métaux labiles par les disques chélatants : validation de la méthode et application sur des matrices environnementales. Rapport de stage de master 2. Université Paris-Est Créteil Val-de-Marne.

BREssy A. (2010) - Flux de micropolluants dans les eaux de ruissellement urbaines. Effets de différents modes de gestion des eaux pluviales. Thèse de doctorat, Université Paris Est, Ecole des Ponts ParisTech.

Chojnacka K. (2010) - Biosorption and bioaccumulation - the prospects for practical applications. Environment International. 36, 3 : 299-307

Clozel B., Ruban V., Durand C., Conil P. (2006) - Origin and mobility of heavy metals in contaminated sediments from retention and infiltration ponds. Applied Geochemistry. 21(10) : 1781-1798

El Mufleh A., Bechet B., Ruban V. (2010) — Etude des phases porteuses des polluants métalliques dans des sédiments de bassins d'infiltration des eaux pluviales. Actes du congrès Novatech Lyon.

Flores-Rodriguez J. (1992) — Les métaux toxiques dans les eaux pluviales en milieu urbain: caractéristiques physico-chimiques. Thèse de doctorat, Université Paris-Val-de-Marne.

Gourlay-France C., Delmas F., Mazzella N., Tusseau-Vuillemin M-H. (2010) — Que sait-on de la biodisponibilité des contaminants dissous dans le milieu aquatique ? Sciences Eaux \& Territoires IRSTEA. 1 : 6-11

Hossain M.A., Furumai H., NaKajima F., Aryal R.K. (2007) Heavy metals speciation in soakaways sediment and evaluation of metal retention properties of surrounding soil. Water Science and Technology. 56(11) : 81-89

Inventaire National Du Patrimoine Naturel. (2006) Caractéristique de la zone Natura 2000. Consultable en ligne: http://inpn.mnhn.fr/site/natura2000/FR1112013

Karlsson K., Viklander M. (2008) - Trace Metal Composition in Water and Sediment from Catch Basins. Journal of Environmental Engineering. 134(10) : 870-878

Karlsson K., Viklander M., Scholes L., Revitt M. (2010) Heavy metal concentrations and toxicity in water and sediment from stormwater ponds and sedimentation tanks. Journal of Hazardous Materials. 178(1-3) : 612-618

Lee P-K., Touray J-C., Baillif P., Ildefonse J-P. (1997) - Heavy metal contamination of settling particles in a retention pond along the A-71 motorway in Sologne, France. The Science of the Total Environment. 201(1) : 1-15

Ministere De L'ecologie, Du Developpement Durable Et De L'ENERGIE (MEDDE) (2012) - Évaluation de l'état des eaux de surface continentales (cours d'eau, canaux, plans d'eau). Guide technique. MEDDE

Smolyakov B.S., Ryzhikh A.P., Romanov R.E. (2010) - The fate of $\mathrm{Cu}, \mathrm{Zn}$, and $\mathrm{Cd}$ in the initial stage of water system contamination: The effect of phytoplankton activity. Journal of Hazardous Materials. 184(1-3) : 819-825

STEAD-DEXTER K., WARD N.I. (2004) — Mobility of heavy metals within freshwater sediments affected by motorway stormwater. Science of the total environment. 334-335 : 271-277

Stu - Agences De L'Eau. (1994) - Guide technique des bassins de retenue d'eaux pluviales. Technique \& Documentation Lavoisier.

TAssin B., Mouchel J.M., Aires N. (2004) - A posteriori analysis of the design and the maintenance of retention ponds in the Ile-de-France region. Actes du congrès Novatech. Lyon

Tran Khac V., Vinçon-Leite B., Varrault G., Lemaire B. J., TAssin B., NAscimento N. (2012) - Urban lakes : interaction between phytoplankton dynamics and trace metal speciation. 12th WWW YES, Arcueil, France

VArrault G., Rocher V., Bracmort G., Louis Y., Matar Z. (2012) - Vers une nouvelle méthode de détermination des métaux labiles dans les milieux aquatiques. Techniques sciences et méthodes. 4 : 56-66

Weiss J.D., Hondzo M., Semmens M. (2006) - Storm Water Detention Ponds: Modeling Heavy Metal Removal by Plant Species and Sediments, Journal of environmental engineering. Journal of Environmental Engineering. 132, 9 : 1034-1042

Yousef Y.A., BAKer D.M., Hvitved-Jacobsen T. (1996) Modelling and impact of metal accumulation in bottom sediments of wet ponds. The Science of the Total Environment. 189-190 : 349-354 